\title{
Effect of bottle gourd leaf (Lagenaria siceraria) extract on the quality of
} beef meatball

\author{
NA Saba, MA Hashem*, MAK Azad, MA Hossain and M Khan
}

Department of Animal Science, Faculty of Animal Husbandry, Bangladesh Agricultural University, Mymensingh2202, Bangladesh

\begin{abstract}
The experiment was conducted to find out the effect of different levels of bottle gourd leaf extraction on beef meatballs. Ground beef samples were divided into four treatment groups having bottle gourd leaf extracts as control $\mathrm{T}_{1}(0 \%), \mathrm{T}_{2}(1 \%), \mathrm{T}_{3}(2 \%), \mathrm{T}_{4}(3 \%)$. Proximate analysis, sensory tests (color, flavor, tenderness, juiciness, and overall acceptability), cooking loss, pH value, free fatty acids (FFA), thiobarbituric acid values (TBARS), peroxide value (POV) and microbiological examination were determined. Days of intervals of experiment were $0,15^{\text {th }}, 30^{\text {th }}$ and $45^{\text {th }}$ days. Data were analyzed using SAS Statistical software. DM content of all the treatment groups differ significantly $(p<0.05)$. In contrast, DM content increased significantly $(p<0.05)$ with the advancement of different days of intervals. The CP and EE content at different treatment levels differ significantly $(p<0.05)$. The FFA, TBARs, POV values were decreased significantly $(p<0.05)$. The color, flavor, tenderness, juiciness, overall acceptability, raw and cooked $\mathrm{pH}$ were increased at different treatment levels significantly $(p<0.05)$. The cooking loss $(\%)$ with the advancement of days of intervals were differ significantly $(p<0.05)$. TCC $(\log$ CFU/g) and TYMC $(\log C F U / g)$ were decreased significantly $(p<0.05)$ at different treatment levels. Parameters studied showed that, $2 \%$ bottle gourd leaf extract added meat balls were the best quality, so it may be concluded that $2 \%$ bottle gourd leaf extract can be used in beef meatball as a source of antioxidant and antimicrobial agent to increase shelf-life of meatballs.
\end{abstract}

Key words: bottle gourd leaf extracts, quality, shelf-life, anti oxidant, beef meatball

Bangladesh Animal Husbandry Association. All rights reserved.

Bang. J. Anim. Sci. 2018. 47 (2):105-113

\section{Introduction}

Beef is the largest source of meat in Bangladesh. Meatball is processed comminuted meat and is very popular among some countries within the Asian region. Meatball is ground or minced meat rolled into a small ball, sometimes along with other ingredients, such as bread/biscuit crumbs, minced onion, eggs, butter and seasonings. Many non-meat ingredients are also added to meatball to increase their nutritional and functional value. Meat is a biologically complex and model systems have been used in an attempt to gain a fundamental understanding of the lipid and protein oxidation processes (Lee et al., 2003). Lipid oxidation and bacterial contamination are the main factors that determine food quality loss and shelf-life reduction. Therefore, delaying lipid oxidation and preventing bacterial crosscontamination are highly relevant to food processors (Fernández-López et al. 2005). Oxidative processes in meat lead to the degradation of lipids and proteins which, in turn, contribute to the deterioration in flavor, texture and color of displayed meat products (Decker et al., 1995). To prevent the autoxidation process antioxidants have been used for many years (Lahucky et al., 2010). It has also been reported that these natural antioxidants, especially of plant source, have greater application potential for consumer's acceptability, palatability, stability and shelf-life of meat products (Jung et al., 2010). One such plant with a potential to be used as an antioxidant is bottle gourd (Lagenaria siceraria) leaf extracts. The plant known as bottle gourd is a common vegetable used throughout Bangladesh. Therefore, there has been a growing interest in natural ingredients to be used in food and food products as preservatives instead of synthetic chemicals. Consequently, search for natural additives, especially of plant origin, has notably increased in recent years (Naveena et al, 2008). Changes in consumer's demand of meat products as well as increased global competition are causing an unprecedented spur in processing and ingredient system developments within the meat manufacturing sector.

*Corresponding author: hashem_mdabul@yahoo.com 
No study yet been conducted on the extract of bottle gourd leaf in meat products. Therefore, the aim of proposed study is to investigate the suitable amount of bottle gourd leaf extract for beef meatball formulation and effect on the quality. Hence, objectives of the study was to examine the addition of bottle gourd leaf extracts on sensory, proximate, physicochemical, biochemical, microbiological analysis and recommend value added beef meatball enriched with bottle gourd leaf extracts.

\section{Materials and Methods}

\section{Meat sample collection}

Boneless beef of $2.5 \mathrm{~kg}$ from freshly slaughtered cattle and bottle gourd leaf extract were collected from a local market named K.R market at BAU campus, Mymensingh. The meat sample was immediately transferred to the "Animal Science Laboratory" in Bangladesh Agricultural University, Mymensingh.

\section{Preparation of jar and other instruments}

All necessary instruments and jars or containers were cleaned with hot water and detergent powder then dried properly before starting the experimental activities.

\section{Sample preparation}

About $2.5 \mathrm{~kg}$ of fresh beef sample was taken for the preparation of beef meatball. First the beef was properly cleaned with fresh water and the fat was trimmed of with sharp knife. Then the beef was grinded properly and the spices, salt, ice flakes, refined vegetable oil, refined bottle gourd leaf extracts, sauce was mixed with the grinded beef properly as per experimental design. There were four treatment groups treated as control group $\left(T_{1}\right), 1 \%$ bottle gourd leaf extract $\left(T_{2}\right), 2 \%$ bottle gourd leaf extracts $\left(T_{3}\right)$, and $3 \%$ bottle gourd leaf extract as $\left(T_{4}\right)$. Then beef meatball of proper shape was prepared separately. It was then boiled in hot water for 2-3 minutes. Then the water was removed from the meatball properly and was fried in hot oil until reddish brown color was obtained.

\section{Sensory evaluation}

Different sensory attributes were examined. Each meatball sample was evaluated by a trained 8-member panel. The sensory questionnaires measured intensity on a 5-point balanced semantic scale (weak to strong) for the attributes color, smell, tenderness, juiciness, and overall acceptability. Eight training sessions were held to familiarize the judges with the attributes to be evaluated and the scale to be used (Rubio et al., 2007). Prior to sample evaluation, all panelists participated in orientation sessions to familiarize with the scale attributes (color, smell, juiciness, tenderness, overall acceptability) of beef meatball using intensity scale. All samples were served in the Petri dishes. Sensory evaluation was accomplished at 0 day and repeated at 15, 30 and 45 day.

\section{Proximate composition}

Proximate composition on Dry Matter, Ether Extract, Crude Protein and Ash were carried out according to the methods (AOAC, 2016).

\section{Physicochemical properties measurement}

\section{Raw and cooked pH measurement}

$\mathrm{pH}$ value of raw and cooked meatball was measured using $\mathrm{pH}$ meter from raw meatball homogenate. It was prepared by blending $5 \mathrm{~g}$ of meat with $10 \mathrm{ml}$ distilled water.

\section{Biochemical analysis}

There were three types of biochemical analysis Viz. free fatty acid, peroxide value, thiobarbituric acid value. Free fatty acid value was determined according to Rukunudin et al., (1998). Peroxide value was determined according to Sallam et al., (2004). Lipid oxidation was assessed in triplicate using the 2thiobarbituric acid method described by Schmedes and Holmer (1989).

\section{Microbial assessment}

For microbial assessment total viable count, coliform count and yeast-mould count was undertaken. A quantity of $10 \mathrm{~g}$ beef meatball sample was aseptically excised from stored stock sample. Each of the stored beef meatball samples was thoroughly and uniformly macerated in a mechanical blender using sterile diluents $(0.1 \%$ peptone water $)$ as per recommendation of International Organization for Standardization (ISO, 1995). A quantity of ten gram of the minced meat meatball sample was taken aseptically transferred into a sterile container containing $90 \mathrm{ml}$ of $0.1 \%$ peptone water. A homogenized suspension was made in a sterile blender. Thus 1:10 dilution of the samples was obtained. Later on using whirly mixture machine different serial dilutions ranging from $10^{-2}$ to $10^{-6}$ were prepared. Microbiological analyses were determined by Ikhlas et al., (2012). 


\section{Statistical analysis}

Data were analyzed using $4 \times 4$ factorial experiment in completely randomized design replicated three times per cell using SAS 9.1.3 version Statistical Discovery software, NC, USA. Duncan's Multiple Range Test (DMRT) test was used to determine the significance of differences among treatments means.

\section{Results and Discussion}

\section{Sensory evaluation}

From Table 1 it was found that the sensory quality after fortification with bottle gourd leaf extracts was deteriorated with increased storage period. The range of overall observed color score at different treatment was 3.50 to 4.42 , flavor score was 3.75 to 4.50 , tenderness score was 3.25 to 4.33 , juiciness score was 3.25 to 4.17 and overall acceptability score was 3.50 to 4.00 . The range of different day's intervals of overall observation of overall acceptability score was 2.75 to 4.58 . Among four treatments most preferable color, flavor, tenderness and juiciness was observed from $2 \%$ bottle gourd leaf extract group. The present findings is in agreement with Gonzalez et al., (2008) where he stated that dried plum ingredients in raw and precooked pork sausage negatively affect sensory attributes viz. color, texture and flavor as well as the nutritional quality of the product.

\section{Proximate analysis}

From Table 2 the DM content was increased with the increased storage period because moisture loss was decreased with storage period. The range of overall observed DM content at different treatments was 44.45 to $46.45 \%$. The range of overall observation of different days of interval DM content was 42.68 to $48.09 \%$. Among four treatments most preferable DM content was observed from $\mathrm{T}_{4}$ (3\%) bottle gourd leaf extract). The highest amount of DM content indicates this product is less preferable. DM increased for the moisture loss of beef meatball samples with the advance of storage time. The most preferable DM content was observed from 0 day and less preferable DM content from $45^{\text {th }}$ day. Similar results were reported for Indonesian traditional meatballs with a moisture content ranged from 69.52 to $71.17 \%$ (Purnomo and Rahardiyan 2008). Devatkal et al., (2010) reported that incorporation pomegranate rind and seed powder extracts did not affect the DM content of goat meat patties. The range of overall observed moisture at different treatments was 43.61 to $49.18 \%$. The CP content was decreased with the increased storage period. The range of overall observed $\mathrm{CP}$ content at different treatments was 22.35 to $23.17 \%$. Among three treatments most preferable CP content was observed from $2 \%$ bottle gourd leaf extract group. The range of overall observed of different days of intervals of CP content was 22.21 to $23.43 \%$. The range of overall observed EE content at different treatments was 11.37 to $12.15 \%$. The range of overall observed of different days of intervals of EE content was 11.35 to $12.21 \%$. Bottle gourd leaf extracts contains higher amount of EE than control group. The Malaysian Food Regulation of 1985 stated that manufactured meat should not contain more than $30 \%$ fat. Malaysian beef meatballs can be classified as low-fat meatballs since the fat content ranges from 1.69 to 11.09 . The range of overall observed ash content at different treatments was 1.29 to $2.41 \%$. The range of overall observed of different days of intervals of ash content was 1.56 to $1.79 \%$. The ash content was significantly changed with the increased storage period. The same trend was also observed by Konieczny et al., (2007) and they reported that ash content increased during frozen storage which is related to our findings.

\section{Physicochemical properties}

From Table 3 the range of overall observed raw $\mathrm{pH}$ at different treatments was 5.69 to $5.80 \%$. Among four treatments most preferable raw $\mathrm{pH}$ was observed from $3 \%$ bottle gourd leaf extract group. The highest amount of raw $\mathrm{pH}$ indicates this product is most preferable for consumers' health. The raw $\mathrm{pH}$ was decreased with the increased storage period. Bacteria and mold have a tendency to increase with increasing storage time, and they secrete components that affect the increasing raw $\mathrm{pH}$. Choi et al., (2009) reported that meat batter containing dietary fiber from rice bran have higher $\mathrm{pH}$ values. The data showed a slight decrease in the raw $\mathrm{pH}$ values and an increase in the acidity values for all samples along with storage time during the 45 days of storage as a result of increased free fatty acids due to rancidity. The data showed a slight increase in the cooked $\mathrm{pH}$ values and a decrease in the acidity values for all samples along with natural antioxidants as a result of the decrease of free fatty acids due to lower rancidity. The range of overall observed cooked $\mathrm{pH}$ at different treatments was 5.89 to 6.10 . 
Bottle gourd leaf extract on meatball

Table 1: Effects of bottle gourd leaf extract on sensory parameters in beef meatball (Values are means \pm SD )

\begin{tabular}{|c|c|c|c|c|c|c|c|c|c|}
\hline \multirow{2}{*}{$\begin{array}{l}\text { Param } \\
\text { eters }\end{array}$} & \multirow[t]{2}{*}{ DI } & \multicolumn{4}{|c|}{ Treatments } & \multirow[t]{2}{*}{ Mean } & \multicolumn{3}{|c|}{ Level of significance } \\
\hline & & $\mathbf{T}_{1}$ & $\mathbf{T}_{\mathbf{2}}$ & $\mathbf{T}_{\mathbf{3}}$ & $\mathbf{T}_{4}$ & & Treat. & DI & T*DI \\
\hline \multirow{5}{*}{ Color } & 0 & $4.00 \pm 0.00$ & $4.67 \pm 0.33$ & $5.00 \pm 0.00$ & $4.33 \pm 0.33$ & $4.50^{a} \pm 0.16$ & \multirow{5}{*}{0.0013} & \multirow{5}{*}{$<.0001$} & \multirow{5}{*}{0.9001} \\
\hline & 15 & $4.33 \pm 0.33$ & $4.67 \pm 0.33$ & $4.67 \pm 0.33$ & $4.33 \pm 0.33$ & $4.53^{a} \pm 0.33$ & & & \\
\hline & 30 & $3.33 \pm 0.33$ & $3.67 \pm 0.33$ & $4.33 \pm 0.33$ & $3.67 \pm 0.33$ & $3.75^{\mathrm{b}} \pm 0.33$ & & & \\
\hline & 45 & $2.33 \pm 0.33$ & $3.33 \pm 0.33$ & $3.67 \pm 0.33$ & $3.00 \pm 0.00$ & $3.08^{\mathrm{c}} \pm 0.25$ & & & \\
\hline & Mean & $3.50^{c} \pm 0.25$ & $4.08^{\mathrm{ab}} \pm 0.33$ & $4.42^{a} \pm 0.25$ & $3.83^{b c} \pm 0.25$ & & & & \\
\hline \multirow{5}{*}{ Flavor } & 0 & $4.33 \pm 0.33$ & $5.00 \pm 0.00$ & $5.00 \pm 0.00$ & $4.00 \pm 0.00$ & $4.58^{a} \pm 0.08$ & \multirow{5}{*}{0.0003} & \multirow{5}{*}{$<.0001$} & \multirow{5}{*}{0.7244} \\
\hline & 15 & $4.33 \pm 0.33$ & $4.67 \pm 0.33$ & $4.67 \pm 0.33$ & $4.33 \pm 0.33$ & $4.50^{a} \pm 0.33$ & & & \\
\hline & 30 & $3.67 \pm 0.33$ & $4.33 \pm 0.33$ & $4.67 \pm 0.33$ & $3.67 \pm 0.33$ & $3.75^{\mathrm{b}} \pm 0.33$ & & & \\
\hline & 45 & $2.33 \pm 0.33$ & $3.33 \pm 0.33$ & $3.67 \pm 0.33$ & $3.00 \pm 0.00$ & $3.08^{\mathrm{c}} \pm 0.25$ & & & \\
\hline & Mean & $3.67^{b} \pm 0.33$ & $4.33^{a} \pm 0.25$ & $4.50^{a} \pm 0.25$ & $3.75^{\mathrm{b}} \pm 0.16$ & & & & \\
\hline \multirow{5}{*}{$\begin{array}{l}\text { Tendern } \\
\text { ess }\end{array}$} & 0 & $4.33 \pm 0.33$ & $4.67 \pm 0.33$ & $5.00 \pm 0.00$ & $4.00 \pm 0.00$ & $4.50^{a} \pm 0.16$ & \multirow{5}{*}{$<.0001$} & \multirow{5}{*}{$<.0001$} & \multirow{5}{*}{0.9571} \\
\hline & 15 & $4.00 \pm 0.00$ & $4.33 \pm 0.33$ & $4.67 \pm 0.33$ & $3.33 \pm 0.33$ & $4.08^{b} \pm 0.25$ & & & \\
\hline & 30 & $3.33 \pm 0.33$ & $3.67 \pm 0.33$ & $4.00 \pm 0.00$ & $3.33 \pm 0.33$ & $3.58^{\mathrm{c}} \pm 0.25$ & & & \\
\hline & 45 & $2.67 \pm 0.33$ & $3.33 \pm 0.33$ & $3.67 \pm 0.33$ & $2.33 \pm 0.33$ & $3.00^{d} \pm 0.33$ & & & \\
\hline & Mean & $3.58^{\mathrm{b}} \pm 0.25$ & $4.00^{a} \pm 0.33$ & $4.33^{a} \pm 0.16$ & $3.25^{\mathrm{b}} \pm 0.25$ & & & & \\
\hline \multirow{5}{*}{$\begin{array}{l}\text { Juicines } \\
\text { s }\end{array}$} & 0 & $4.33 \pm 0.33$ & $4.67 \pm 0.33$ & $5.00 \pm 0.00$ & $4.00 \pm 0.00$ & $4.50^{a} \pm 0.16$ & \multirow{5}{*}{.0001} & \multirow{5}{*}{$<.0001$} & \multirow{5}{*}{0.9571} \\
\hline & 15 & $3.67 \pm 0.33$ & $4.67 \pm 0.33$ & $4.67 \pm 0.33$ & $3.67 \pm 0.33$ & $4.17^{a} \pm 0.33$ & & & \\
\hline & 30 & $3.33 \pm 0.33$ & $3.67 \pm 0.33$ & $3.67 \pm 0.33$ & $3.00 \pm 0.00$ & $3.42^{b} \pm 0.25$ & & & \\
\hline & 45 & $2.33 \pm 0.33$ & $3.00 \pm 0.00$ & $3.33 \pm 0.33$ & $2.33 \pm 0.33$ & $2.75^{c} \pm 0.25$ & & & \\
\hline & Mean & $3.42^{\mathrm{b}} \pm 0.33$ & $4.00^{a} \pm 0.25$ & $4.17^{a} \pm 0.25$ & $3.25^{\mathrm{b}} \pm 0.16$ & & & & \\
\hline \multirow{5}{*}{$\begin{array}{l}\text { Overall } \\
\text { accepta } \\
\text { bility }\end{array}$} & 0 & $4.33 \pm 0.33$ & $4.67 \pm 0.33$ & $5.00 \pm 0.00$ & $4.33 \pm 0.33$ & $4.58^{a} \pm 0.25$ & \multirow{5}{*}{0.0902} & \multirow{5}{*}{$<.0001$} & \multirow{5}{*}{0.7522} \\
\hline & 15 & $4.33 \pm 0.33$ & $4.33 \pm 0.33$ & $4.67 \pm 0.33$ & $4.33 \pm 0.33$ & $4.42^{\mathrm{a}} \pm 0.33$ & & & \\
\hline & 30 & $3.67 \pm 0.33$ & $3.67 \pm 0.33$ & $3.67 \pm 0.33$ & $3.00 \pm 0.00$ & $3.50^{\mathrm{b}} \pm 0.25$ & & & \\
\hline & 45 & $2.67 \pm 0.33$ & $3.33 \pm 0.33$ & $2.67 \pm 0.33$ & $2.33 \pm 0.33$ & $2.75^{\mathrm{c}} \pm 0.33$ & & & \\
\hline & Mean & $3.75^{\mathrm{ab}} \pm 0.33$ & $4.00^{a} \pm 0.33$ & $4.00^{\mathrm{a}} \pm 0.25$ & $3.50^{\mathrm{b}} \pm 0.25$ & & & & \\
\hline
\end{tabular}

Sensory scores were 5 for excellent, 4 for very good, 3 for good, 2 for fair, and 1 for poor. Mean in each row having different superscript varies significantly at values $p<0.05$. $\mathrm{T}_{1}=$ Controlled group, $\mathrm{T}_{2}=1 \%$ Bottle gourd leaf extract, $\mathrm{T}_{3}=2 \%$, Bottle gourd leaf extract, $\mathrm{T}_{4}=3 \%$, Bottle gourd leaf extract, DI $=\mathrm{Day}$ Intervals, Treat $=$ Treatment, $T * D I=$ Interaction of Treatment and Day Intervals. 
Saba et al. (2018) Bang. J. Anim. Sci. 47 (2):105 -113

Table 2: Effect of bottle gourd leaf extract on proximate component in beef meatball (Values are means \pm SD)

\begin{tabular}{|c|c|c|c|c|c|c|c|c|c|}
\hline \multirow{2}{*}{$\begin{array}{l}\text { Parame } \\
\text { ters }\end{array}$} & \multirow[t]{2}{*}{ DI } & \multicolumn{4}{|c|}{ Treatments } & \multirow[t]{2}{*}{ Mean } & \multicolumn{3}{|c|}{ Level of significance } \\
\hline & & $\mathbf{T}_{1}$ & $\mathbf{T}_{2}$ & $\mathbf{T}_{3}$ & $\mathbf{T}_{4}$ & & Treat. & DI & T*DI \\
\hline \multirow{5}{*}{ DM (\%) } & 0 & $43.41 \pm 0.02$ & $43.41 \pm 0.03$ & $41.57 \pm 0.01$ & $42.32 \pm 0.01$ & $42.68^{d} \pm 0.02$ & \multirow{5}{*}{$<.0001$} & \multirow{5}{*}{$<.0001$} & \multirow{5}{*}{$<.0001$} \\
\hline & 15 & $45.18 \pm 0.01$ & $46.97 \pm 0.02$ & $43.76 \pm 0.01$ & $44.11 \pm 0.01$ & $44.68^{c} \pm 0.01$ & & & \\
\hline & 30 & $46.99 \pm 0.01$ & $46.94 \pm 0.01$ & $45.72 \pm 0.01$ & $44.70 \pm 0.01$ & $46.09^{b} \pm 0.01$ & & & \\
\hline & 45 & $49.20 \pm 0.01$ & $48.63 \pm 0.03$ & $47.89 \pm 0.03$ & $46.67 \pm 0.03$ & $48.09^{a} \pm 0.02$ & & & \\
\hline & Mean & $46.19^{b} \pm 0.01$ & $46.45^{a} \pm 0.02$ & $44.73^{c} \pm 0.01$ & $44.45^{d} \pm 0.01$ & & & & \\
\hline \multirow{5}{*}{$\begin{array}{c}\text { Moisture } \\
(\%)\end{array}$} & 0 & $44.34 \pm 0.44$ & $46.81 \pm 0.29$ & $48.27 \pm 0.26$ & $49.41 \pm 0.48$ & $47.20^{b} \pm 0.37$ & \multirow{5}{*}{$<.0001$} & \multirow{5}{*}{$<.0001$} & \multirow{5}{*}{$<.0001$} \\
\hline & 15 & $43.64 \pm 0.59$ & $46.97 \pm 0.11$ & $50.03 \pm 0.14$ & $51.71 \pm 0.15$ & $48.09^{a} \pm 0.25$ & & & \\
\hline & 30 & $43.34 \pm 0.06$ & $45.88 \pm .018$ & $48.93 \pm 0.34$ & $47.50 \pm 0.27$ & $46.49^{c} \pm 0.21$ & & & \\
\hline & 45 & $43.14 \pm 0.05$ & $44.71 \pm 0.08$ & $47.65 \pm 0.22$ & $47.83 \pm 0.18$ & $45.83^{d} \pm 0.13$ & & & \\
\hline & Mean & $43.61^{d} \pm 0.28$ & $46.09^{c} \pm 0.16$ & $48.71^{b} \pm 0.24$ & $49.18^{a} \pm 0.27$ & & & & \\
\hline \multirow{5}{*}{$\mathrm{CP}(\%)$} & 0 & $23.41 \pm 0.02$ & $23.42 \pm 0.03$ & $23.41 \pm 0.04$ & $23.48 \pm 0.02$ & $23.43^{\mathrm{a}} \pm 0.03$ & \multirow{5}{*}{$<.0001$} & \multirow{5}{*}{$<.0001$} & \multirow{5}{*}{$<.0001$} \\
\hline & 15 & $23.11 \pm 0.02$ & $22.25 \pm 0.01$ & $23.50 \pm 0.01$ & $23.06 \pm 0.03$ & $22.98^{\mathrm{b}} \pm 0.02$ & & & \\
\hline & 30 & $21.60 \pm 0.01$ & $22.49 \pm 0.02$ & $23.02 \pm 0.05$ & $22.75 \pm 0.01$ & $22.46^{\mathrm{C}} \pm 0.02$ & & & \\
\hline & 45 & $21.28 \pm 0.02$ & $22.25 \pm 0.01$ & $22.75 \pm 0.01$ & $22.55 \pm 0.01$ & $22.21^{d} \pm 0.01$ & & & \\
\hline & Mean & $22.35^{d} \pm 0.02$ & $22.60^{c} \pm 0.02$ & $23.17^{a} \pm 0.03$ & $22.96^{\mathrm{b}} \pm 0.02$ & & & & \\
\hline \multirow{5}{*}{ EE (\%) } & 0 & $12.11 \pm 0.01$ & $11.56 \pm 0.07$ & $12.65 \pm 0.01$ & $12.52 \pm 0.02$ & $12.21^{a} \pm 0.03$ & \multirow{5}{*}{$<.0001$} & \multirow{5}{*}{$<.0001$} & \multirow{5}{*}{$<.0001$} \\
\hline & 15 & $12.02 \pm 0.01$ & $11.47 \pm 0.01$ & $12.54 \pm 0.01$ & $12.41 \pm 0.01$ & $12.11^{\mathrm{b}} \pm 0.01$ & & & \\
\hline & 30 & $11.91 \pm 0.01$ & $11.39 \pm 0.01$ & $11.97 \pm 0.01$ & $12.22 \pm 0.02$ & $11.87^{c} \pm 0.01$ & & & \\
\hline & 45 & $11.53 \pm 0.01$ & $11.06 \pm 0.02$ & $11.45 \pm 0.01$ & $11.37 \pm 0.01$ & $11.35^{\mathrm{d}} \pm 0.01$ & & & \\
\hline & Mean & $11.89^{b} \pm 0.01$ & $11.37^{c} \pm 0.03$ & $12.15^{\mathrm{a}} \pm 0.01$ & $12.13^{a} \pm 0.01$ & & & & \\
\hline \multirow{5}{*}{ Ash (\%) } & 0 & $1.15 \pm 0.01$ & $1.38 \pm 0.01$ & $1.43 \pm 0.01$ & $2.27 \pm 0.01$ & $1.56^{c} \pm 0.01$ & \multirow{5}{*}{$<.0001$} & \multirow{5}{*}{$<.0001$} & \multirow{5}{*}{$<.0001$} \\
\hline & 15 & $1.19 \pm 0.01$ & $1.42 \pm 0.01$ & $1.28 \pm 0.02$ & $2.35 \pm 0.01$ & $1.56^{\mathrm{C}} \pm 0.01$ & & & \\
\hline & 30 & $1.37 \pm 0.01$ & $1.47 \pm 0.01$ & $1.37 \pm 0.01$ & $2.43 \pm 0.01$ & $1.68^{\mathrm{b}} \pm 0.01$ & & & \\
\hline & 45 & $1.45 \pm 0.01$ & $1.67 \pm 0.02$ & $1.47 \pm 0.01$ & $2.60 \pm 0.01$ & $1.79^{\mathrm{a}} \pm 0.01$ & & & \\
\hline & Mean & $1.29^{d} \pm 0.01$ & $1.48^{\mathrm{b}} \pm 0.01$ & $1.38^{\mathrm{c}} \pm 0.01$ & $2.41^{a} \pm 0.01$ & & & & \\
\hline
\end{tabular}

Mean in each row having different superscript varies significantly at values $p<0.05 . T_{1}=$ Controlled group, $\mathrm{T}_{2}=1 \%$ Bottle Gourd leaf extract, $\mathrm{T}_{3}=2 \%$, Bottle gourd leaf extract, $\mathrm{T}_{4}=3 \%$, Bottle gourd leaf extract, $\mathrm{DI}=$ Day Intervals, Treat $=$ Treatment, $T * \mathrm{DI}=$ Interaction of Treatment and Day Intervals.

The highest amount of cooked $\mathrm{pH}$ indicates this product is most preferable for consumers' health than other treatments. The lowest amount of cooking loss indicates this product is most preferable for consumers' choices than other treatments. The range of overall observed of different days of intervals of cooking loss was 26.19 to $22.14 \%$. The cooking loss was decreased with the increased storage period. The less preferable cooking loss was observed from 0 day and maximum cooking loss was observed from $45^{\text {th }}$ day observation. Cooking loss refers to the reduction in weight of meatballs during the cooking process (Jama et al., 2008).
Cooking loss in meat cuts is important for maintaining an attractive retail display of meat. Dietary fiber supplementations increase the bulk and prevent cooking loss in meat products with no / fewer changes in textural parameters by enhancing water binding capabilities and carries great economic advantages for both the consumers and processors. Turhan et al., (2005) reported that addition of hazelnut pellicle fiber was found to be effective in improving cooking yield, dimensional changes and thickness of beef burgers. Cooking yield is an important data that are used by the meat industry to predict the behavior of their products during processing (Ulu, 2006). 


\section{Bottle gourd leaf extract on meatball}

Table 3: Effect of bottle gourd leaf extract on physicochemical parameters in beef meatball (Means \pm SD )

\begin{tabular}{|c|c|c|c|c|c|c|c|c|c|}
\hline \multirow{2}{*}{$\begin{array}{l}\text { Param } \\
\text { eters }\end{array}$} & \multirow[t]{2}{*}{ DI } & \multicolumn{4}{|c|}{ Treatments } & \multirow[t]{2}{*}{ Mean } & \multicolumn{3}{|c|}{ Level of significance } \\
\hline & & $\mathbf{T}_{\mathbf{1}}$ & $\mathbf{T}_{2}$ & $\mathbf{T}_{3}$ & $\mathbf{T}_{4}$ & & Treat. & DI & $\mathbf{T} * \mathbf{D I}$ \\
\hline \multirow{5}{*}{ Raw pH } & 0 & $5.74 \pm 0.04$ & $5.82 \pm 0.01$ & $5.86 \pm 0.01$ & $5.85 \pm 0.01$ & $5.82^{a} \pm 0.02$ & \multirow{5}{*}{$\begin{array}{l}<.000 \\
1\end{array}$} & \multirow{5}{*}{$\begin{array}{l}<.000 \\
1\end{array}$} & \multirow{5}{*}{$\begin{array}{l}0.001 \\
5\end{array}$} \\
\hline & 15 & $5.72 \pm 0.01$ & $5.81 \pm 0.01$ & $5.87 \pm 0.01$ & $5.81 \pm 0.01$ & $5.80^{\mathrm{a}} \pm 0.01$ & & & \\
\hline & 30 & $5.68 \pm 0.01$ & $5.69 \pm 0.01$ & $5.73 \pm 0.01$ & $5.78 \pm 0.01$ & $5.72^{b} \pm 0.01$ & & & \\
\hline & 45 & $5.63 \pm 0.1$ & $5.67 \pm 0.01$ & $5.70 \pm 0.01$ & $5.77 \pm 0.01$ & $5.69^{c} \pm 0.01$ & & & \\
\hline & Mean & $5.69^{c} \pm 0.02$ & $5.75^{\mathrm{b}} \pm 0.01$ & $5.80^{a} \pm 0.01$ & $5.79^{a} \pm 0.01$ & & & & \\
\hline \multirow{5}{*}{$\begin{array}{l}\text { Cooked } \\
\mathrm{pH}\end{array}$} & 0 & $6.06 \pm 0.01$ & $6.09 \pm 0.01$ & $6.13 \pm 0.01$ & $6.20 \pm 0.01$ & $6.12^{\mathrm{a}} \pm 0.01$ & \multirow{5}{*}{$\begin{array}{l}<.000 \\
1\end{array}$} & \multirow{5}{*}{$\begin{array}{l}<.000 \\
1\end{array}$} & \multirow{5}{*}{$\begin{array}{l}<.000 \\
1\end{array}$} \\
\hline & 15 & $5.98 \pm 0.01$ & $6.04 \pm 0.01$ & $6.16 \pm 0.01$ & $6.12 \pm 0.01$ & $6.07^{\mathrm{b}} \pm 0.01$ & & & \\
\hline & 30 & $5.78 \pm 0.01$ & $6.02 \pm 0.01$ & $6.12 \pm 0.01$ & $6.08 \pm 0.01$ & $6.00^{c} \pm 0.01$ & & & \\
\hline & 45 & $5.75 \pm 0.01$ & $5.86 \pm 0.01$ & $5.94 \pm 0.01$ & $5.94 \pm 0.04$ & $5.87^{d} \pm 0.02$ & & & \\
\hline & Mean & $5.89^{d} \pm 0.01$ & $6.00^{c} \pm 0.01$ & $6.10^{b} \pm 0.01$ & $6.07^{a} \pm 0.02$ & & & & \\
\hline \multirow{5}{*}{$\begin{array}{c}\text { Cooking } \\
\text { loss } \\
(\%)\end{array}$} & 0 & $26.73 \pm 0.26$ & $26.08 \pm 0.02$ & $26.03 \pm 0.02$ & $25.95 \pm 0.01$ & $26.19^{a} \pm 0.08$ & \multirow{5}{*}{$\begin{array}{l}<.000 \\
1\end{array}$} & \multirow{5}{*}{$\begin{array}{l}<.000 \\
1\end{array}$} & \multirow{5}{*}{$\begin{array}{l}<.000 \\
1\end{array}$} \\
\hline & 15 & $25.85 \pm 0.08$ & $24.98 \pm 0.06$ & $25.94 \pm 0.03$ & $25.84 \pm 0.01$ & $25.65^{\mathrm{b}} \pm 0.04$ & & & \\
\hline & 30 & $24.43 \pm 0.27$ & $23.17 \pm 0.21$ & $22.63 \pm 0.03$ & $22.07 \pm 0.01$ & $23.07^{c} \pm 0.13$ & & & \\
\hline & 45 & $22.32 \pm 0.55$ & $22.17 \pm 0.02$ & $22.07 \pm 0.01$ & $21.98 \pm 0.03$ & $22.14^{d} \pm 0.15$ & & & \\
\hline & Mean & $24.83^{a} \pm 0.29$ & $24.11^{b} \pm 0.08$ & $24.17^{b} \pm 0.02$ & $23.96^{\mathrm{b}} \pm 0.01$ & & & & \\
\hline
\end{tabular}

Mean in each row having different superscript varies significantly at values $p<0.05$. $T_{1}=$ Controlled group, $\mathrm{T}_{2}=1 \%$ Bottle gourd leaf extract, $\mathrm{T}_{3}=2 \%$, Bottle gourd leaf extract, $\mathrm{T}_{4}=3 \%$, Bottle gourd leaf extract, $\mathrm{DI}=$ Day Intervals, Treat $=$ Treatment, $\mathrm{T} * \mathrm{DI}=$ Interaction of Treatment and Day Intervals.

Table 4 Effect of bottle gourd leaf extract on bio-chemical parameters in beef meatball (Mean \pm SD)

\begin{tabular}{|c|c|c|c|c|c|c|c|c|c|}
\hline \multirow[t]{2}{*}{ Parameters } & \multirow[t]{2}{*}{ DI } & \multirow[b]{2}{*}{$\mathbf{T}_{1}$} & \multicolumn{3}{|c|}{ Treatments } & \multirow[t]{2}{*}{ Mean } & \multicolumn{3}{|c|}{$\begin{array}{c}\text { Level of } \\
\text { significance }\end{array}$} \\
\hline & & & $\mathbf{T}_{2}$ & $\mathbf{T}_{\mathbf{3}}$ & $\mathbf{T}_{4}$ & & $\begin{array}{l}\mathbf{T} \\
\mathbf{r} \\
\mathbf{e} \\
\mathbf{a} \\
\mathbf{t} .\end{array}$ & $\begin{array}{l}\text { D } \\
\text { I }\end{array}$ & $\begin{array}{l}\mathbf{T} * \mathbf{D} \\
\mathbf{I}\end{array}$ \\
\hline \multirow{5}{*}{ FFA (\%) } & 0 & $0.31 \pm 0.003$ & $0.31 \pm 0.01$ & $0.29 \pm 0.01$ & $0.28 \pm 0.003$ & $0.29^{d} \pm 0.006$ & \multirow{5}{*}{$\begin{array}{l}< \\
\dot{0} \\
0 \\
0 \\
1\end{array}$} & \multirow{2}{*}{$<$} & \multirow{5}{*}{$\begin{array}{c}0.00 \\
19\end{array}$} \\
\hline & 15 & $0.34 \pm 0.002$ & $0.33 \pm 0.003$ & $0.32 \pm 0.004$ & $0.30 \pm 0.004$ & $0.32^{c} \pm 0.003$ & & & \\
\hline & 30 & $0.38 \pm 0.001$ & $0.35 \pm 0.001$ & $0.34 \pm 0.002$ & $0.32 \pm 0.006$ & $0.35^{\mathrm{b}} \pm 0.002$ & & $\begin{array}{l}0 \\
0\end{array}$ & \\
\hline & 45 & $0.42 \pm 0.004$ & $0.41 \pm 0.001$ & $0.40 \pm 0.01$ & $0.36 \pm 0.004$ & $0.39^{\mathrm{a}} \pm 0.004$ & & 0 & \\
\hline & Mean & $0.36^{a} \pm 0.002$ & $0.35^{\mathrm{b}} \pm 0.004$ & $0.34^{c} \pm 0.006$ & $0.32^{d} \pm 0.004$ & & & 1 & \\
\hline \multirow{5}{*}{$\mathrm{PV}(\mathrm{meq} / \mathrm{kg})$} & 0 & $4.11 \pm 0.01$ & $3.97 \pm 0.01$ & $4.03 \pm 0.003$ & $4.03 \pm 0.03$ & $4.03^{d} \pm 0.01$ & \multirow{5}{*}{$\begin{array}{l}< \\
. \\
0 \\
0 \\
0 \\
1\end{array}$} & \multirow{5}{*}{$\begin{array}{l}< \\
0 \\
0 \\
0 \\
0 \\
1\end{array}$} & \multirow{5}{*}{$\begin{array}{c}<.00 \\
01\end{array}$} \\
\hline & 15 & $4.16 \pm 0.01$ & $4.04 \pm 0.01$ & $4.11 \pm 0.01$ & $4.09 \pm 0.01$ & $4.10^{c} \pm 0.01$ & & & \\
\hline & 30 & $4.32 \pm 0.02$ & $4.09 \pm 0.01$ & $4.23 \pm 0.01$ & $4.15 \pm 0.01$ & $4.20^{\mathrm{b}} \pm 0.01$ & & & \\
\hline & 45 & $4.53 \pm 0.01$ & $4.45 \pm 0.01$ & $4.37 \pm 0.01$ & $4.28 \pm 0.02$ & $4.40^{a} \pm 0.01$ & & & \\
\hline & Mean & $4.28^{a} \pm 0.01$ & $4.14^{\mathrm{c}} \pm 0.01$ & $4.18^{\mathrm{b}} \pm 0.01$ & $4.14^{c} \pm 0.02$ & & & & \\
\hline \multirow{5}{*}{$\begin{array}{c}\text { TBARS } \\
(\mathrm{mg}-\mathrm{MA} / \mathrm{kg})\end{array}$} & 0 & $0.41 \pm 0.001$ & $0.40 \pm 0.01$ & $0.39 \pm 0.001$ & $0.39 \pm 0.002$ & $0.39^{d} \pm 0.003$ & \multirow{5}{*}{$\begin{array}{l}< \\
\dot{0} \\
0 \\
0 \\
1\end{array}$} & $<$ & \multirow{5}{*}{$\begin{array}{c}<.00 \\
01\end{array}$} \\
\hline & 15 & $0.48 \pm 0.01$ & $0.43 \pm 0.002$ & $0.41 \pm 0.002$ & $0.40 \pm 0.001$ & $0.43^{c} \pm 0.004$ & & $\dot{0}$ & \\
\hline & 30 & $0.55 \pm 0.001$ & $0.50 \pm 0.001$ & $0.49 \pm 0.001$ & $0.49 \pm 0.001$ & $0.51^{b} \pm 0.001$ & & $\begin{array}{l}0 \\
0\end{array}$ & \\
\hline & 45 & $0.76 \pm 0.001$ & $0.58 \pm 0.001$ & $0.58 \pm 0.001$ & $0.59 \pm 0.001$ & $0.63^{a} \pm 0.001$ & & 0 & \\
\hline & Mean & $0.55^{a} \pm 0.003$ & $0.48^{b} \pm 0.003$ & $0.47^{c} \pm 0.001$ & $0.47^{d} \pm 0.001$ & & & 1 & \\
\hline
\end{tabular}

Mean in each row having different superscript varies significantly at values $p<0.05$. $\mathrm{T}_{1}=$ Controlled group, $\mathrm{T}_{2}=1 \%$ Bottle gourd leaf extract, $\mathrm{T} 3=2 \%$, Bottle gourd leaf extract, $\mathrm{T}_{4}=3 \%$, Bottle gourd leaf extract, $\mathrm{DI}=$ Day Intervals, Treat $=$ Treatment, $T * \mathrm{DI}=$ Interaction of Treatment and Day Intervals. 
Saba et al. (2018) Bang. J. Anim. Sci. 47 (2):105 -113

Table 5: Effect of bottle gourd leaf extract on different microbial population in beef meatball (Mean \pm SD)

\begin{tabular}{|c|c|c|c|c|c|c|c|c|c|}
\hline \multirow[t]{2}{*}{ Parameters } & \multirow[t]{2}{*}{ DI } & \multicolumn{4}{|c|}{ Treatments } & \multirow[t]{2}{*}{ Mean } & \multicolumn{3}{|c|}{ Level of significance } \\
\hline & & $\mathbf{T}_{1}$ & $\mathbf{T}_{\mathbf{2}}$ & $\mathbf{T}_{\mathbf{3}}$ & $\mathbf{T}_{4}$ & & Treat. & DI & $\mathbf{T} * \mathbf{D I}$ \\
\hline \multirow{5}{*}{$\begin{array}{l}\text { TVC } \\
(\log C F U / g)\end{array}$} & 0 & $4.62 \pm 0.01$ & $4.54 \pm 0.01$ & $4.73 \pm 0.005$ & $4.36 \pm 0.01$ & $4.49^{d} \pm 0.008$ & \multirow{5}{*}{$<.0001$} & \multirow{5}{*}{$<.0001$} & \multirow{5}{*}{$<.0001$} \\
\hline & 15 & $4.84 \pm 0.02$ & $4.69 \pm 0.003$ & $4.71 \pm 0.01$ & $4.69 \pm 0.006$ & $4.74^{c} \pm 0.01$ & & & \\
\hline & 30 & $4.94 \pm 0.02$ & $4.76 \pm 0.01$ & $4.75 \pm 0.007$ & $4.74 \pm 0.009$ & $4.80^{\mathrm{b}} \pm 0.01$ & & & \\
\hline & 45 & $5.43 \pm 0.05$ & $5.14 \pm 0.01$ & $5.18 \pm 0.003$ & $5.13 \pm 0.003$ & $5.22^{a} \pm 0.02$ & & & \\
\hline & Mean & $4.96^{a} \pm 0.02$ & $4.78^{\mathrm{b}} \pm 0.01$ & $4.76^{\mathrm{b}} \pm 0.006$ & $4.73^{c} \pm 0.007$ & & & & \\
\hline \multirow{5}{*}{$\begin{array}{l}\text { TCC } \\
(\log C F U / g)\end{array}$} & 0 & $1.15 \pm 0.01$ & $1.14 \pm 0.01$ & $1.19 \pm 0.01$ & $1.15 \pm 0.006$ & $1.16^{\mathrm{a}} \pm 0.01$ & \multirow{5}{*}{$<.0001$} & \multirow{5}{*}{$<.0001$} & \multirow{5}{*}{$<.0001$} \\
\hline & 15 & $1.12 \pm 0.004$ & $1.12 \pm 0.003$ & $1.15 \pm 0.003$ & $1.11 \pm 0.001$ & $1.13^{b} \pm 0.003$ & & & \\
\hline & 30 & $1.11 \pm 0.004$ & $1.05 \pm 0.01$ & $1.04 \pm 0.006$ & $1.02 \pm 0.003$ & $1.06^{\mathrm{c}} \pm 0.006$ & & & \\
\hline & 45 & $1.03 \pm 0.004$ & $1.02 \pm 0.004$ & $0.98 \pm 0.006$ & $0.95 \pm 0.004$ & $0.99^{d} \pm 0.004$ & & & \\
\hline & Mean & $1.10^{\mathrm{a}} \pm 0.005$ & $1.08^{c} \pm 0.006$ & $1.09^{\mathrm{b}} \pm 0.006$ & $1.06^{\mathrm{d}} \pm 0.003$ & & & & \\
\hline \multirow{5}{*}{$\begin{array}{l}\text { TYMC } \\
(\log C F U / g)\end{array}$} & 0 & $1.95 \pm 0.02$ & $1.86 \pm 0.007$ & $1.86 \pm 0.006$ & $1.85 \pm 0.001$ & $1.88^{\mathrm{a}} \pm 0.008$ & \multirow{5}{*}{$<.0001$} & \multirow{5}{*}{$<.0001$} & \multirow{5}{*}{$<.0001$} \\
\hline & 15 & $1.74 \pm 0.003$ & $1.55 \pm 0.01$ & $1.61 \pm 0.002$ & $1.56 \pm 0.01$ & $1.62^{b} \pm 0.006$ & & & \\
\hline & 30 & $1.55 \pm 0.02$ & $1.38 \pm 0.009$ & $1.41 \pm 0.01$ & $1.38 \pm 0.008$ & $1.43^{c} \pm 0.01$ & & & \\
\hline & 45 & $1.13 \pm 0.006$ & $1.05 \pm 0.007$ & $1.12 \pm 0.005$ & $1.10 \pm 0.004$ & $1.10^{\mathrm{d}} \pm 0.005$ & & & \\
\hline & Mean & $1.59^{\mathrm{a}} \pm 0.01$ & $1.46^{\mathrm{c}} \pm 0.008$ & $1.50^{\mathrm{b}} \pm 0.006$ & $1.47^{c} \pm 0.006$ & & & & \\
\hline
\end{tabular}

Mean in each row having different superscript varies significantly at values $p<0.05$. $\mathrm{T}_{1}=$ Controlled group, $\mathrm{T}_{2}=1 \%$ Bottle gourd leaf extracts, $\mathrm{T}_{3}=2 \%$, Bottle gourd leaf extract, $\mathrm{T}_{4}=3 \%$, Bottle gourd leaf extracts, DI=Day Intervals, Treat $=$ Treatment, $T^{*} \mathrm{DI}=$ Interaction of Treatment and Day Intervals.

The values of cooking yield were similar to the results in high-fat Kung-wan meatballs reported by (Huang et al., 2005). The cooking yield of the Kung-Wan significantly decreased with higher natural antioxidant extract levels (Hsu and Sun, 2006). The range of overall observed cooking loss at different treatments was 24.83 to $23.96 \%$.

\section{Biochemical properties}

From Table 4 shows that the range of overall observed peroxide value at different treatment levels was 4.28 to $4.14 \%$. Throughout the storage time, peroxide values were generally higher in control samples than in others. The range of overall observed of different days of intervals of FFA was 0.29 to $0.39 \%$. The range of overall observed FFA value at different treatments was 0.36 to $0.32 \%$. The range of overall observed of different days of intervals of peroxide value was 4.03 to $4.40 \%$.
Meat with higher lipid oxidation values also showed higher protein oxidation greater metmyoglobin formation. Lund et al., (2007) reported on the peroxide values in sausage with three treatments (rosemary extract, collagen fiber preparation impregnated with rosemary extract and collagen hydrolyses impregnated with rosemary extract); samples with three treatments showed lower values than the control. The peroxide value of plain meat loaf was $0.38,1.33$ and 2.40 at 0,3 and 6 days, respectively, in aerobic storage at $4^{\circ} \mathrm{C}$. Natural antioxidants, in particular polyphenols, are the major plant compounds which have the ability to attenuate the oxidative damage of a tissue indirectly by enhancing natural defenses of cell directly by scavenging the free radical species combat pathological disorders generated by physicochemical Reactive Oxygen Species (ROS) (Du et al., 2010). Rhee and Myers (2003) examined peroxide values in plain meat loaf made from ground goat meat and reported 
a similar trend in peroxide value during storage. Generally, TBA levels significantly $(p<0.05)$ increased with storage time, showing decreasing shelf life. The range of overall observed of different days of intervals of TBA value was 0.39 to $0.63 \%$. Among these four treatments most preferable TBA value was observed from $2 \%$ and $3 \%$ bottle gourd leaf extract group. The lowest amount of TBA value indicates this product is most preferable for consumers' health. The results of this study confirm that treated bottle gourd leaf extract meatball can delay lipid oxidation significantly, reducing the potential risk induced by lipid oxidation. The results of this study confirm that $1 \%, 2 \%, 3 \%$ bottle gourd leaf extracts can delay lipid oxidation significantly, reducing the potential risk induced by lipid oxidation.

\section{Microbiological assessment}

Effects of bottle gourd leaf extract on different microbial population in beef meatballs have shown in Table 5.TVC of beef meatballs ranges from 4.96-4.73 (logCFU/g) at different treatment levels. Among four treatments, the TVC in the control sample (4.96 logs CFU/g) was significantly higher than in the samples treated with $1 \%, 2 \%$, and $3 \%$ of bottle gourd leaf extracts. The less amount of TVC value indicates this product is most preferable for consumers' health. The range of TVC value was 5.22 to $4.49(\log \mathrm{CFU} / \mathrm{g})$ for days of intervals. During storage TVC value was increased with increasing of storage life. The range of TCC of beef meatballs was 1.10 to $1.06(\log C F U / g)$, at different treatment levels. The range of overall observed TYMC of beef meatballs was 1.59 to $1.46(\log \mathrm{CFU} / \mathrm{g})$ at different treatment levels. The range of TYMC value was 1.88 to 1.10 $(\log C F U / g)$ due to different days of intervals. Similar values for TVC, TCC and TYMC also found in value added beef meat balls (Siddiqua et al., 2018). Fernández-López et al., (2003) reported on the results of a research study related to antimicrobials in beef meatballs. They reported that the presence of mold and yeasts were not detected in any cooked meatball samples. The initial value of TVC for fresh beef was $5.12 \log \mathrm{CFU} / \mathrm{g}$ beef, indicating good quality beef.

\section{Conclusion}

In conclusion, sensory, proximate, biochemical and microbiological studies shows that $2 \%$ bottle gourd leaf extract shown better performance for beef meatball preservation. Addition of $2 \%$ bottle gourd leaf extract had the highest tenderness, overall acceptability, raw and cooked $\mathrm{pH}$, DM, Ash, POV and TBARs. So, it may be concluded that $2 \%$ bottle gourd leaf extract can be used in beef meatball as a source of antioxidant and antimicrobial agent to increase shelf-life of meatballs.

\section{Acknowledgement}

The financial support of the Bangladesh Agricultural University, Mymensingh is greatly appreciated and the authors are grateful to the Department of Animal Science of Bangladesh Agricultural University for providing essential supports for conducting the research.

\section{References}

AOAC (2016). Official Methods of Analysis. (20 th Edition) Association of Official Analytical Chemists. Washington, USA.

Choi YS, H Doo-Jeong, K Mi-Ai, L Hack-Young, J Hyun-Wook, $\mathrm{K}$ Jong-youn and $\mathrm{KJ}$ cheon (2009). Characteristics of low -fat meat emulsion systems with pork fat replaced by vegetable oils and rice bran fiber. Meat Science 82(2):266-271.

Devatkal SK, K Narsaiah and A Borah (2010). Antioxidant effect of extracts of kinnow rind, pomegranate rind and seed powders in cooked goat meat patties. Meat Science 85:155-159.

Du J, Y Zeng Wang, Y Qian, $\mathrm{H} \mathrm{Li}$, QW Chen and J Cui (2010). Cu Zn SOD gene expression and its relationship with anti-oxidative capacity and pork quality. South African Journal of Animal Science 40:265-272.

Fernandez- Lopez J, J M Fernandez- Gines, L Aleson- Carbonell, E Sayas- Barberia, E Sendra, and JA Perez- Alvarez (2003). Functional components from citrus by products and their application in meat products. Trend in Food science and technology 15:176 -185.

Fernández-López, J, N Zhi, L Aleson- Carbonell, JA Pérez and $V$ Kun (2005). Antioxidant and antibacterial activities of natural extracts: Application in beef. Meat Science 69(3):371380.

Gonzalez MTN, RM Baleman, RK Miller, JT Keeton and KS Rhee (2008). Antioxidant properties of dried plum ingredients in raw and precooked pork sausage. Journal of Food Science 73(50):63-71.

Hsu SY and LY Sun (2006). Comparison on 10 non- meat protein fat substitutes for low-fat Kung-Wans. Journal of Food Engineering 74:47-53.

Huang SC, CY Shiau, TE Liu, CL Chu and DF Hwang (2005). Effects of rice bran on sensory and physicochemical properties of emulsified of pork meatball. Meat Science 70:613- 619 . 
Saba et al. (2018) Bang. J. Anim. Sci. 47 (2):105-113

Ikhlas B, 1 Huda and N Ismail (2012). Effect of cosmos caudatus, polygonum minus and $\mathrm{BHT}$ on physical properties, oxidative process, and microbiological growth of quail meatball during refrigeration storages. Journal of Food Processing and Preservation 36:55-66.

ISO (1995). International Organization for Standardization, Recommendation of the meeting of the subcommittee, on meat and meat products. ISO/TC-36/SC-6. The Netherlands. 10-18.

Jama N, V Muchenje, M Chimonyo, PE Strydom, KD zama and JG Raats (2008). Cooking loss components of beef from Nguni, Bonsmara and Angus steers. African Journal of Agricultural Research 3(6):416-420.

Jung $\mathrm{S}$, J Choe, B Kim, $\mathrm{H}$ Yun, ZA Kruk and $C$ Jo (2010). Effect of dietary mixture of gallic acid and linoleic acid on ant oxidative potential and quality of breast meat from broilers. Meat Science 86:520-526.

Konieczny P, J Stangiershi and J Kijowski (2007). Physical and chemical characteristics and acceptability of home style beef jerky. Meat Science 76:253-257.

Lahucky R, L Nuernberg Kovac, O Bucko and G Nuernberg (2010). Assessment of the antioxidant potential of selected plant extracts in vitro and in vivo experiments on pork. Meat Science 85:779-784.

Lee S, AL Phillips, DC Liebler and C Faustman (2003). Porcine oxymyoglobin and lipid oxidation in vitro. Meat Science 63:241-247.

Lund MN, MS Hviid and LH Skibsted (2007). The combined effect of antioxidants and modified atmosphere packaging on protein and lipid oxidation in beef patties during chill storage. Meat Science 76:226-233.
Naveena BM, AR Sen, S Vaithiyanathan, Y Babji and N Kondaiah (2008). Comparative efficacy of pomegranate juice, pomegranate rind powder extract and BHT as antioxidants in cooked chicken patties. Meat Science 80:1304-1308

Purnomo A and D Rahardiyan (2008). Indonesian traditional meatball: Review article Int. Food Research journal 15:101-108.

Rubio B, B Martinej, MJ Sinchey, MD Garciacachan, J Rovira, and Jaime (2007). Study of self-life of a dry fermented sausage Salchichon made from raw material enriched in monounsaturated and polyunsaturated fatty acids and stored under modified atmospheres. Meat Science 76:128-137.

Rhee KS and CE Myers (2003). Sensory properties and lipid oxidation in aerobically refrigerated cooked ground goat meat. Meat Science 66: 189-194.

Rukunudin IH, PJ White, CJ Bern and TB Bailey (1998). A modified method for determining free fatty acids from small soybean sample sizes. Journal of American Oil Chemistry Society 75:563-568.

Sallam KI, M Ishioroshi and K Samejima (2004). Antioxidants and antimicrobial effects of garlic in chicken sausage. Lebensm Wiss Technology 37:849-855.

Schemedes A and G Holmer (1989). A new Thiobarbituric acid (TBA) method for determining free malondialdehyde and hydroperoxides selectively as a measure of lipid peroxidation. Journal of African Oil Chemistry Society 66:813-817. 